\title{
A Google Trends-based approach for monitoring NSSI
}

This article was published in the following Dove Press journal:

Psychology Research and Behavior Management

12 December 2013

Number of times this article has been viewed

\author{
Nicola Luigi Bragazzi \\ DINOGMI, Department of \\ Neuroscience, Rehabilitation, \\ Ophthalmology, Genetics, Maternal \\ and Child Health, Section of \\ Psychiatry, University of Genoa, \\ Genoa, Italy
}

\begin{abstract}
Non-suicidal self-injury (NSSI) is an intentional, direct, and socially unacceptable behavior resulting in the destruction of one's own body tissues with no intention of dying or committing suicide, even though it is associated with a higher risk of attempted, planned, or just considered suicide. In this preliminary report, we introduce the concept of "NSSI 2.0"; that is to say, the study of the Internet usage by subjects with NSSI, and we introduce a Google Trends-based approach for monitoring NSSI, called NSSI infodemiology and infoveillance. Despite some limitations, Google Trends has already proven to be reliable for infectious diseases monitoring, and here we extend its application and potentiality in the field of suicidology. Ad hoc web portals and surveys could be designed in light of the reported results for helping people with NSSI.
\end{abstract}

Keywords: infodemiology, infoveillance, Internet, non-suicidal self-injury

\section{Introduction NSSI 2.0}

Non-suicidal self-injury (NSSI) is a deliberate and direct behavior, not conforming to social, cultural, and religious norms, and resulting in the destruction of one's own body tissues. It is direct since indirect forms of procuring self-harm like substance use, eating disorders, and heavy drinking are not considered as NSSI. It is deliberate since it is intentional, voluntary, and consists of an action or a series of actions carried out with no intention of death or committing suicide, ${ }^{1}$ even though NSSI is associated with a higher risk of considered or attempted suicide. ${ }^{2}$ Some studies have shown that NSSI is able to predict suicidal behaviors, and that subjects engaged in NSSI are seven times more likely to report making at least one suicidal gesture; ten times more likely to report making at least one suicide attempt; three times more likely to report having thought of suicide; and six times more likely to report having planned it. ${ }^{3}$ Other studies have replicated this association between NSSI and suicidal tendencies, such as in the prospective survey performed by Guan et $\mathrm{al}^{4}$ or in the study conducted by Nock et $\mathrm{al}^{5}$ who found that over $70 \%$ of adolescent self-injurers reported at least one lifetime suicide attempt and 55\% of them multiple attempts. Moreover, they discovered that this particular subgroup of NSSI injurers was characterized by a longer history of NSSI, no experience and/or feelings of pain during the injurious episodes, and a higher heterogeneity in practicing it. For these reasons, Joiner speculated that NSSI injurers were initially frightened by the idea of suicide and only after multiple attempts would they become more competent and confident with it. ${ }^{6} \mathrm{~A}$ longer history of NSSI could be
Correspondence: Nicola Luigi Bragazzi School of Public Health, Department of Health Sciences (DISSAL), University of Genoa, Via Antonio Pastore I, I6I32 Genoa, Italy Tel +390103537664 Fax +390103537669 Email robertobragazzi@gmail.com 
considered a good predictor of suicide attempts. On the other hand, NSSI could function as a strategy for avoiding suicide, such as a "replacement action" or a substitute for suicide." However, the precise nature of the relationship between NSSI and suicidality remains speculative and unclear.

Even though NSSI is not defined on the basis of its frequency, reiterated and repeated episodes of NSSI are more likely to follow the first episode (reinforcing theory). ${ }^{7}$ Common examples and modalities of NSSI behaviors include self-cutting (generally on the inside part of the arms), scratching, scraping, burning, bruising, biting, pinching, breaking bones, skin picking, hair pulling/ripping, interfering with wound healing, embedding objects, carving words and/or symbols, needlesticking, hitting walls, and banging/punching themselves and objects. ${ }^{7-11}$ More than half of self-injurers make use of multiple methods ${ }^{10}$ and, as already mentioned, this heterogeneity in practicing NSSI is related with a higher risk of long-term NSSI.

NSSI is a highly prevalent disorder, having an estimated lifetime prevalence among adolescents varying from 5.5\% to $30.7 \%$, while the 12 -month prevalence rates vary from $7.5 \%$ to $37.2 \%$, and the 6 -month prevalence rates vary from $13.9 \%$ to $16.3 \%,{ }^{12}$ while among adults, lifetime prevalence is computed to be $5.9 \%$ with a 12 -month prevalence rate of $0.9 \%{ }^{10}$

NSSI has, therefore, an important impact on society and represents a huge burden in terms of epidemiology, clinical relevance, and economic costs. NSSI is thus an important public health concern. Costs can be direct and include costs for health care delivery (such as psychopharmacological treatment; psychotherapy; and management of wounds, cuts, burns, or bruises); and indirect such as those due to morbidity, decreased productivity at work, school absence, impairment in social life because of damaged or lost relationships, and self-conscious negative feelings and emotions such as guilt, shame, and embarrassment. ${ }^{13,14}$

Even if there is a lack of an unambiguous and consistent terminology (many synonyms exist such as deliberate selfinjury, deliberate self-harm, non-suicidal self-mutilation, and self-wounding, with slight differences) $)^{7-8,11}$ and NSSI remains an "umbrella term" rather than a precise self-defined nosological entity, ${ }^{15}$ and despite many efforts of providing a systematic and comprehensive consensus (it is worth mentioning the "Classification of Self-Injurious thoughts and behaviors"), ${ }^{16}$ interest in exploring NSSI is increasing in the scientific community, a growing body of evidence is accumulating, and proposals for including NSSI in the forthcoming Diagnostic Statistic Manual (DSM) V version have been put forward, ${ }^{17-20}$ even though in the version released it has not been included. However, NSSI has been at least partially incorporated in the third section of the DSM-V entitled "Emerging measures and models" for diagnoses warranting attention and further research or higher clinical exposure and validation. ${ }^{21-23}$ Besides NSSI, the newly included diagnoses are attenuated psychosis syndrome, depressive episodes with short-duration hypomania, persistent complex bereavement disorder, caffeine use disorder, Internet gaming disorder, neurobehavioral disorder due to prenatal alcohol exposure, and suicidal behavior disorder. Currently, according to the DSM-IV TR and ICD-10, a description of NSSI-like behaviors constitutes one of the items of borderline personality disorder, and NSSI-like symptoms can be found in people with anxiety and mood disorders. However, NSSI has peculiar characteristics that enable it to be distinguished from other mental diseases, since the definition of NSSI as a cluster of symptoms characterizing another psychiatric nosological entity is rather limiting, both from a clinical and a research point of view. Pervasive developmental disorders, as well as other pervasive pathologies should be indeed ruled out in the diagnosis of NSSI. ${ }^{1}$ These peculiar forms and modalities of procuring self-harm in the context of an already extant psychiatric pathology should be termed self-injurious behaviors and should be kept distinct from NSSI.

The precise etiology of NSSI is unknown, but a genetic predisposition and biological impairments, such as alterations of brain morphology, of lipid plasma levels, or of neurotransmitters (in particular, serotoninergic, dopaminergic, and opioid systems), ${ }^{24-26}$ as well as a history of child trauma and exposure to violence, ${ }^{27}$ parent loss, neglect, and sexual abuse/ assault, have been reported. ${ }^{28}$ In childhood trauma survivors, NSSI would have four functions: to re-experience the original trauma and to convey feelings and emotions about it ("sorting out reality" function); ${ }^{29}$ to try to establish a communication with the outer world and to share the need of being heard and understood; to organize the "Self"; and to fight against dissociative processes and trends. However, not all the individuals with a history of traumatic experiences will engage in NSSI behaviors, and it has been suggested that psychiatric traumatic symptoms could provide this missing link. ${ }^{27}$ As far as sexual abuse is concerned, the evidence is mixed and not quite robust, as other scholars have failed to replicate this association and the overall effect could be weak as proven by a meta-analysis that found a mean weighted aggregate phi $=0.23 .^{30}$

NSSI 2.0 is the variety of online activities carried out virtually by NSSI injurers, ranging from the mere consultation of NSSI-related websites to the creation of dedicated portals 
enabling video uploading, chatting and commenting, and the establishment of virtual communities. NSSI 2.0 is the flow of NSSI-related information and materials among the users.

In this research article, we intend to investigate a part of this virtual reality; that is to say, the NSSI queries trends and patterns.

\section{Infodemiology and infoveillance}

Traditional epidemiological surveys suffer from many drawbacks, such as the long term of data retrieval, collection, processing, and elaboration. Moreover, the design of a large multicentric study is complex and time-demanding/timeconsuming. To overcome these hurdles, "infodemiology" and "infoveillance" are new emerging concepts introduced by Gunther Eysenbach when elaborating a promising approach for public health and epidemiological research, ${ }^{31,32}$ based on large-scale monitoring and data-mining, within the conceptual framework of e-health and health Web 2.0. These last terms indicate new modalities, strategies, and practices of health care delivery that exploit, and are supported by, electronic processes and communication, and by a more and more dynamic and interactive information reality (which is the meaning of Web 2.0) in which the user is at the same time a consumer and a producer (that is to say, a prosumer) of the Web content. Within e-health, the patient is at the center of the health care processes, as they are more involved and informed of the many steps of medical decision making.

Infodemiology and infoveillance study all the virtual activities carried out by lay people while surfing health-related sites and communicating and sharing their health status. The main advantage of these new approaches is real time tracking, monitoring, and data collection and analysis.

Both survey-based and experimental studies have shown how people engaging in NSSI behaviors are likely to use more online sites as a preferential platform for conveying their feelings and emotions, and, moreover, the onset age of NSSI coincides with the peak of Internet use and accessing. ${ }^{33}$

Self-injury is fairly prevalent in the general adult population (up to a rate of $4 \%-5.9 \%$, according to Briere and Gil) ${ }^{9}$ and is particularly common in adolescent (up to $46 \%) .{ }^{34}$ The observed mean age of onset is in the range of $13-16$ years. ${ }^{11}$

It is, therefore, of vital clinical importance and relevance that the psychiatrists become aware of the NSSI-related online material and check its reliability in order to be able to react to it and discuss it with their patients. ${ }^{35-39}$ The new media can have a dangerous epidemic and "viral" contagious effect on vulnerable subjects, ${ }^{39-46}$ and some scholars talk about a so-called "virtual cutting edge" (the ways adolescents use the Internet for conveying their feelings and NSSI-related experiences), ${ }^{37}$ a "Werther effect" (that is to say, the effect of improper and inappropriate media reporting of suicide, especially among celebrities and stars, on the lay population), ${ }^{44}$ or a "cyber-suicide phenomenon" (that is to say, a suicide attempt first announced on Internet websites, such as forums, blogs, or chats, which is later followed by the fatality). ${ }^{45}$ NSSI 2.0, being interactive, dynamic, and increasingly expanding, is a complex reality where websites could have a negative effect of reinforcement, ${ }^{38,39,44,45}$ or, on the contrary, could be used for healthier purposes, such as health education and self-managing and coping strategy. Therefore, monitoring the hit searches could act as an efficient real time surveillance system. ${ }^{47,48}$

Google Trends (Google Inc., Menlo Park, CA, USA) is an online tracking system that enables a visualization of hit-search volumes throughout the years, from 2004 on, and a Google-based approach is particularly useful to monitor especially infectious diseases epidemics. ${ }^{49}$ However, its enormous potentiality and applications in other fields of medicine, such as the field of neurology ${ }^{50}$ or suicidology, have been noticed only recently. Sueki ${ }^{51}$ found that the "suicide" search volumes did not correlate with the suicide statistics, but, after introducing a time lag of 1-3 months, "depression" hit-search could be used as a proxy in alerting the monitoring authorities, the surveillance institutions, and the public health officials. Hagihara et $\mathrm{al}^{52}$ interestingly found that searching for "hydrogen sulfide" was associated with the suicide rate in Japan. Other searched terms correlated with "suicide" or "attempted suicide" and the scholars were even able to find age correlations, despite the anonymous nature of the users. Yang et $\mathrm{a}^{53}$ confirmed these findings in the People's Republic of China, while Page et $\mathrm{al}^{54}$ failed to replicate the results in Australia. Gunn and Lester ${ }^{55}$ surveyed suicides using search engines and found that suicide rates in the 50 US states positively correlated with the "suicide" hit search. So far, to our knowledge, no one apart from Sueki ${ }^{56}$ has explored the possibility of monitoring NSSI via Google.

\section{Materials and methods}

Google Trends, an online tracking system of Internet hitsearch volumes that recently merged with its sister project Google Insights for Search (Google Inc.), was searched for the years 2004 to 2012 (as data before 2004 were not available) for "autolesionismo" (Italian for NSSI). Autocorrelation is the correlation of a parameter with itself 
over time. Autocorrelation functions of this series of data, both crude and adjusted partial, were computed using $\mathrm{R}$ environment (freely available at http://www.r-project.org/). ${ }^{57} \mathrm{~A}$ list of NSSI-related terms was searched in Italian and their flux volumes were correlated with the NSSI hit-search data. Pearson's coefficient was calculated with SPSS software V21.0.0 package (IBM Corporation, Armonk, NY, USA) and using R environment. ${ }^{58} P$-value was computed with SPSS and values $\leq 0.05$ were considered statistically significant.

\section{Results}

The pattern of Internet search volume interestingly revealed a cyclic trend and a regular pattern (as can be seen from Figures 1 and 2). The autocorrelation reported statistically significant scores (Figure 2). The most NSSI-related search terms were NSSI and cutting, NSSI and children, NSSI and forums, and causes and reasons of NSSI (Figure 1).

The Italian towns with the higher searching fluxes and volumes were Naples, Milan, Padua, Bari, Bologna, Rome, Palermo, Turin, and Florence; that is to say, we did not observe any geographical gradient between North and South (Figure 1 and Table 1). The queries volumes shown in Figure 1 are normalized values (rather than being the crude absolute values). This finding only appears to be in disagreement with data obtained from surveys, as we monitored the overall aggregate data, without taking into account sex or socioeconomic variables, which undoubtedly contribute to the North-South divide. The Pearson's correlation coefficients (reported in Table 2 and plotted in Figure 3) were all statistically significant

\section{Discussion}

It is difficult to say whether NSSI is an increasing phenomenon or not because of the inconsistency of terminology and the heterogeneity of used methods and approaches, together with the lack of vast and rigorous epidemiological data. However, there is the suspicion that it is increasing, and this is probably due also to the reinforcement provided by the same digital technologies. Traditional epidemiological surveys and techniques are expensive and time consuming,
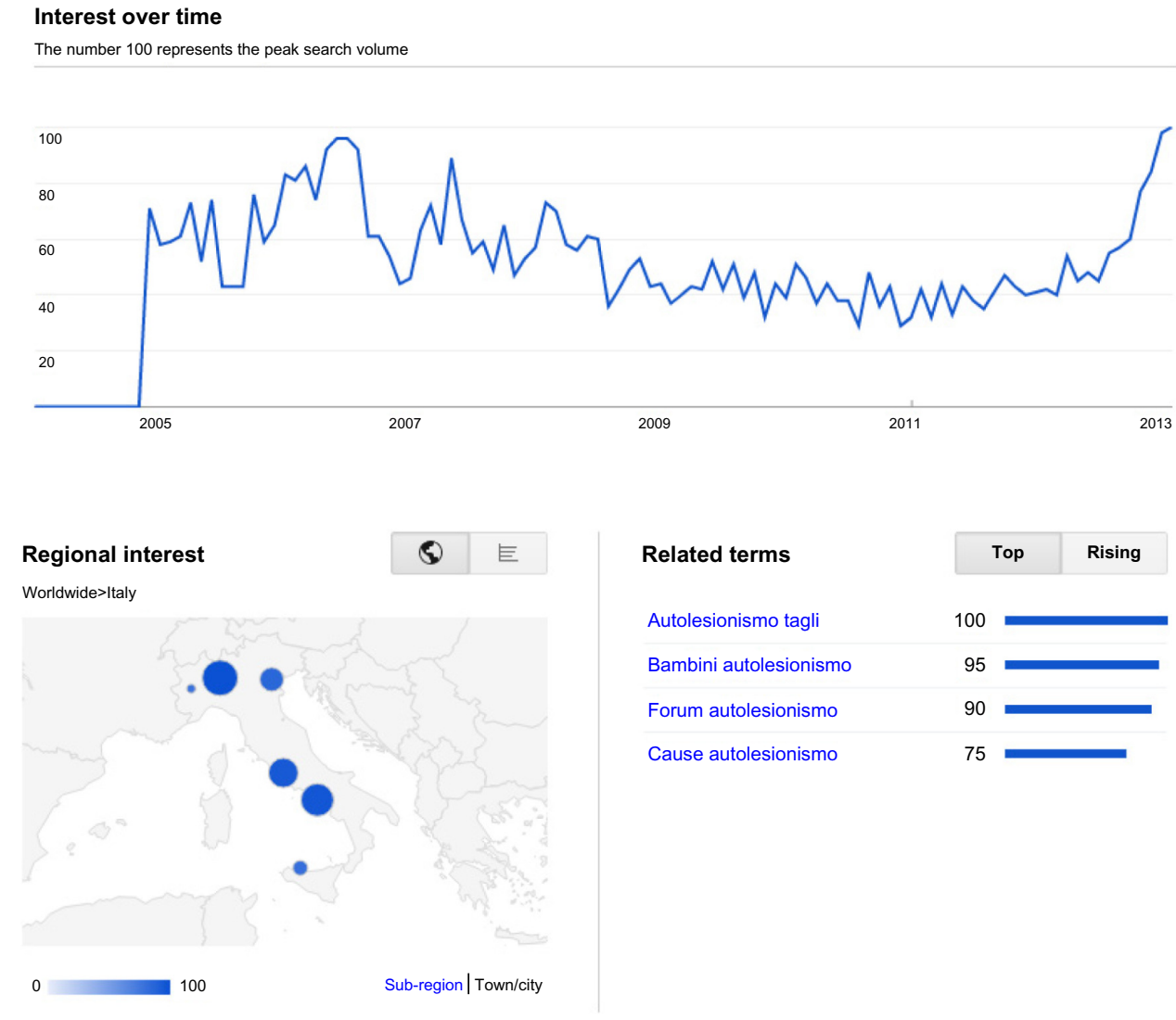

Figure I Google Trends-based NSSI hit-search volume over the years (from 2004 to 20I2).

Notes: NSSI hit-search volume over the years 2004-20I2 (top), a map of Italian towns with higher flux volumes (bottom, left), and a list of the most searched NSSI-related terms (bottom, right).

Abbreviation: NSSI, non-suicidal self-injury 
Autocorrelation

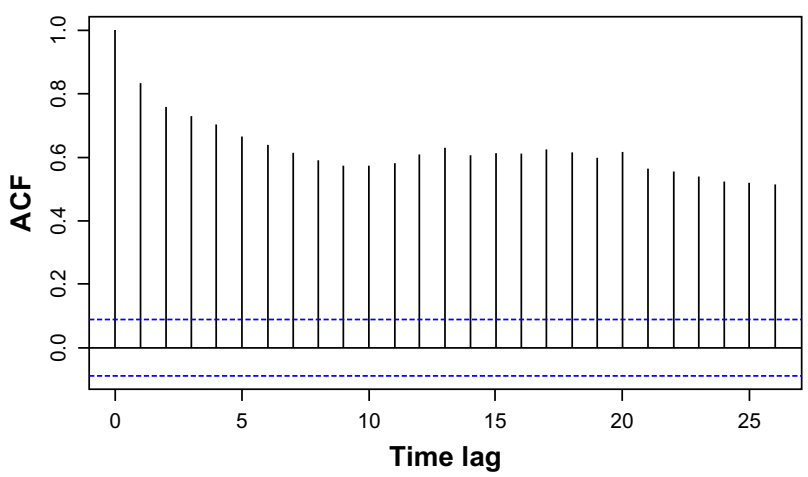

Partial autocorrelation

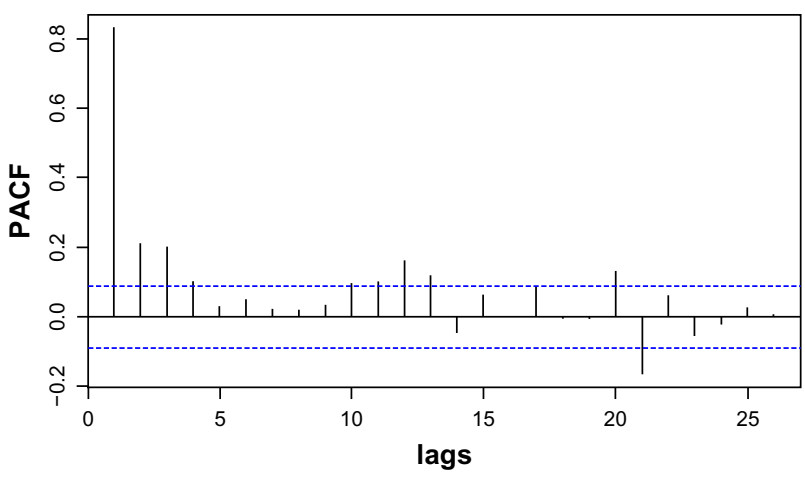

Figure 2 Autocorrelation plot for the NSSI hit-search and partial autocorrelation plot, showing a regular cyclical pattern.

Abbreviations: ACF, autocorrelation function; NSSI, non-suicidal self-injury; PACF, Partial autocorrelation function.

while in this preliminary report we show the feasibility of a Google Trends-based approach, which has been statistically validated.

However, this study has several limitations. Complete epidemiological data for NSSI are not available or are largely incomplete and, for this reason, a Pearson's correlation between NSSI online hit-search volume and NSSI rate could not be performed. Larger multicentric surveys should

Table I A list of the towns with higher normalized NSSI hitsearch flux volumes

\begin{tabular}{ll}
\hline $\begin{array}{l}\text { Towns with high hit } \\
\text { search volumes }\end{array}$ & $\begin{array}{l}\text { Normalized hit search } \\
\text { volume (\%) }\end{array}$ \\
\hline Milan & 100 \\
Naples & 96 \\
Rome & 94 \\
Padua & 86 \\
Palermo & 80 \\
Turin & 78 \\
\hline
\end{tabular}

Abbreviation: NSSI, non-suicidal self-injury.
Table 2 Pearson's correlation coefficients for different hit search volumes related to NSSI and their statistical significance according to their two-tailed $P$-value

\begin{tabular}{lll}
\hline $\begin{array}{l}\text { NSSI-related } \\
\text { hit search }\end{array}$ & $\begin{array}{l}\text { Pearson's correlation } \\
\text { coefficient }\end{array}$ & $\begin{array}{l}P \text {-value } \\
\text { (two tails) }\end{array}$ \\
\hline NSSI and suicide & $r=0.26$ & $P=0.00 * *$ \\
NSSI and blood & $r=0.33$ & $P=0.00 * *$ \\
NSSI and cutting & $r=0.48$ & $P=0.00 * *$ \\
NSSI and razor & $r=0.52$ & $P=0.00 * *$ \\
NSSI and depression & $r=-0.60$ & $P=0.00 * *$ \\
NSSI and anxiety & $r=0.18$ & $P=0.00 * *$ \\
NSSI and bullying & $r=0.12$ & $P=0.01 *$ \\
NSSI and anorexia & $r=-0.23$ & $P=0.00 * *$ \\
NSSI and bulimia & $r=-0.56$ & $P=0.00 * *$ \\
\hline
\end{tabular}

Notes: *Statistically significant, with $P$-value $<0.05$; **statistically significant, with $P$-value $<0.01$.

Abbreviation: NSSI, non-suicidal self-injury.

be carried out in order to provide the researcher with more robust data and correlations.

A second limitation is due to the ambiguity of the search keywords, as also pointed out by other researchers. ${ }^{33}$ Moreover, Google Trends monitors only the queries carried out using Google as a search engine, while other engines do exist (such as Yahoo, MSN, Bing, and so on). On the other hand, Google is the most used search engine worldwide.

A third limitation of this study is that it explores only a part of the multitude of the online NSSI-related activities (such as video uploading and sharing, chatting, and blogging) and, in particular, it investigates the NSSI-related queries patterns and trends documented in the literature. For these reasons, the approach we introduced should be complemented (rather than replaced or substituted) by further studies concerning, for example, the content of Internet websites.

Caution should therefore be taken when interpreting our findings, which are limited to a particular sector of the Internet and should not be generalized to other Internet NSSIrelated activities. Furthermore, the relationship between online and offline activities could, of course, not be addressed or envisaged by such approaches.

Moreover, using search term trends is not intended as a proxy for self-injury prevalence, as individuals could come to search for self-injury for all sorts of reasons, and not simply because they self-injure. Parents, professionals, academics, journalists, and those that are just interested in or curious about NSSI search for NSSI-related terms. The use of Google Trends is intended to stimulate and foster research in the field; not to replace the need for real individual level research.

Google Trends may provide interesting geographic-level data, as found here in various Italian provinces, and this may 


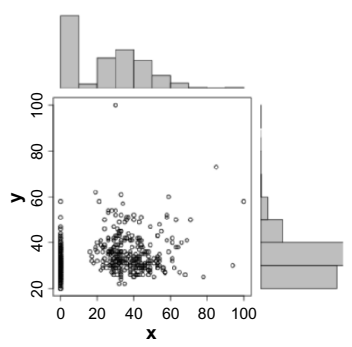

NSSI and suicide

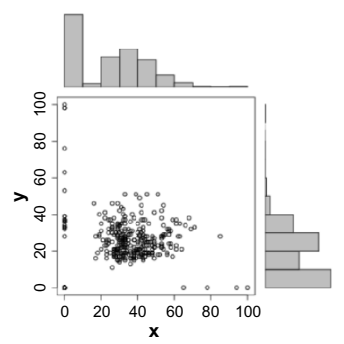

NSSI and razor

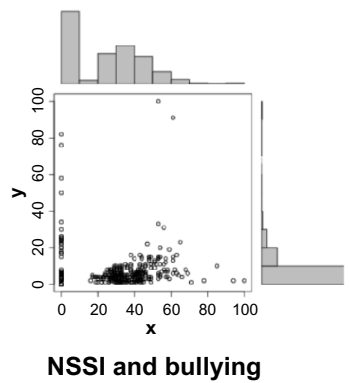

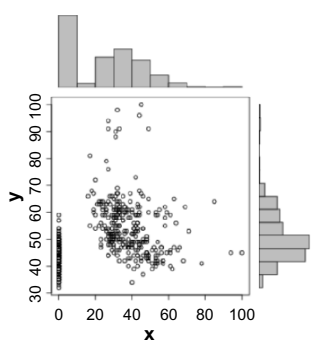

NSSI and blood

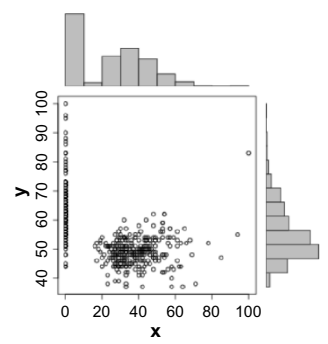

NSSI and depression

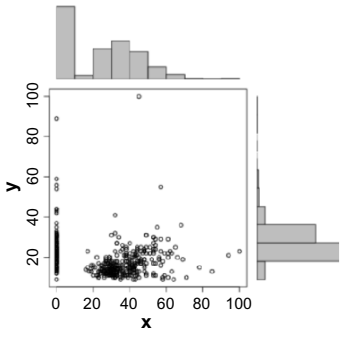

NSSI and anorexia

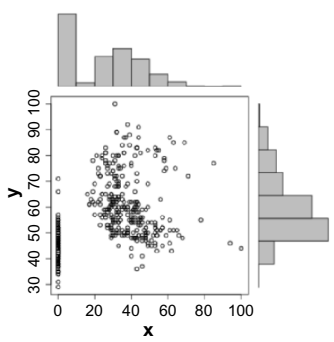

NSSI and cutting

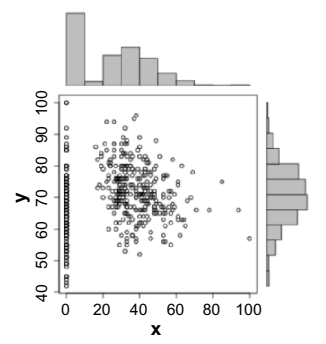

NSSI and anxiety

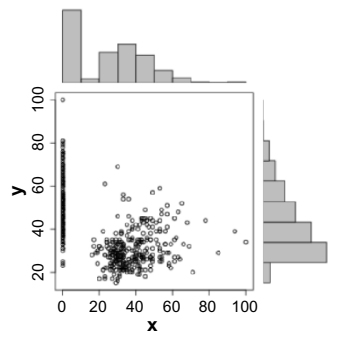

NSSI and bulimia

Figure 3 Pearson's correlation plots for some of the most important NSSI-related hit search terms. Abbreviation: NSSI, non-suicidal self-injury.

provide some insight as to where NSSI is of particular interest due to increased awareness, an actual surge in cases, or a particularly high-profile case that generated local interest. For these reasons, it should be complemented and validated using traditional surveillance measures.

However, this study was one of the first surveys to explore the feasibility of an infoveillance-based approach for detecting the proxies of NSSI behaviors. This could foster further research - also replicating the findings in other regions, and other languages/cultures - and help epidemiologists to design ad hoc surveys and, above all, to help public health officials to implement dedicated web portals in order to meet with patients needs and doubts.

\section{Conclusion}

Despite its limitations, this preliminary exploration showed that a web-based approach for monitoring NSSI is feasible, and that the advantages of this type of approach are varied. Classical statistics are delivered and processed with a delay of some months or, in the worst cases, years, while an infoveillance approach could be able to capture real time online data. Also, ad hoc web portals and surveys could be designed in light of the reported results, also with the aim of developing and implementing specific treatment plans and interventions for adolescent NSSI injurers. Moreover, since scholars have already established a clinical link between NSSI and suicidality, research about the relationship between NSSI and suicide queries should be conducted on Google Trends.

\section{Disclosure}

The author reports no conflicts of interest in this work.

\section{References}

1. Nock MK, Favazza AR. Nonsuicidal self-injury: Definition and classification. In Nock MK, editor. Understanding Nonsuicidal Self-Injury: Origins, Assessment, and Treatment. Washington (DC): American Psychological Association; 2009:9-18.

2. Lofthouse N, Yager-Schweller J. Nonsuicidal self-injury and suicide risk among adolescents. Curr Opin Pediatr. 2009;21(5):641-645. 
3. Whitlock J, Knox KL. The relationship between self-injurious behavior and suicide in a young adult population. Arch Pediatr Adolesc Med. 2007;161(7):634-640.

4. Guan K, Fox KR, Prinstein MJ. Nonsuicidal self-injury as a time-invariant predictor of adolescent suicide ideation and attempts in a diverse community sample. J Consult Clin Psychol. 2012;80:842-849.

5. Nock MK, Joiner TE, Gordon KH, Lloyd-Richardson E, Prinstein MJ. Non-suicidal self-injury among adolescents: Diagnostic correlates and relation to suicide attempts. Psychiatry Res. 2006;144:65-72.

6. Joiner TE. Why People Die by Suicide. Cambridge (MA): Harvard University Press; 2005.

7. Klonsky ED. The functions of deliberate self-injury: a review of the evidence. Clin Psychol Rev. 2007;27(2):226-239.

8. Klonsky ED. Non-suicidal self-injury: an introduction. J Clin Psychol. 2007;63(11):1039-1043.

9. Briere J, Gil E. Self-mutilation in clinical and general population samples: prevalence, correlates, and functions. Am J Orthopsychiatry. 1998;68(4):609-620.

10. Jacobson CM, Gould M. The epidemiology and phenomenology of nonsuicidal self-injurious behavior among adolescents: a critical review of the literature. Arch Suicide Res. 2007;11(2):129-147.

11. Klonsky ED. Non-suicidal self-injury in United States adults: prevalence, sociodemographics, topography and functions. Psychol Med. 2011;41(9):1981-1986.

12. Muehlenkamp JJ, Claes L, Havertape L, Plener PL. International prevalence of adolescent non-suicidal self-injury and deliberate self-harm. Child Adolesc Psychiatry Ment Health. 2012;6:10.

13. Laye-Gindhu A, Schonert-Reichl KA. Nonsuicidal self-harm among community adolescents: understanding the 'whats' and 'whys' of self-harm. J Youth Adolesc. 2005;34:447-457.

14. You J, Lin MP, Leung F. Functions of nonsuicidal self-injury among Chinese community adolescents. J Adolesc. 2013;36(4):737-745.

15. Cloutier P, Martin J, Kennedy A, Nixon MK, Muehlenkamp JJ. Characteristics and co-occurrence of adolescent non-suicidal selfinjury and suicidal behaviours in pediatric emergency crisis services. J Adolesc. 2010;39:259-269.

16. Nock MK. Self-injury. Annu Rev Clin Psychol. 2010;6:339-363.

17. Shaffer D, Jacobson C. Proposal to the DSM-V Childhood Disorder and Mood Disorder Work Groups to Include Non-Suicidal Self-Injury (NSSI) as a DSM-V Disorder. Columbia University, New York State Psychiatric institute, 2009.

18. De Leo D. DSM-V and the future of suicidology. Crisis. 2011;32:233-239.

19. Plener PL, Kapusta ND, Kölch MG, Kaess M, Brunner R. Non-suicidal selfinjury as autonomous diagnosis - implications for research and clinic of the DSM-5 proposal to establish the diagnosis of Non-suicidal self-injury in adolescents. Z Kinder Jugendpsychiatr Psychother. 2012;40:113-120. German.

20. Zetterqvist M, Lundh LG, Dahlström O, Svedin CG. Prevalence and Function of Non-Suicidal Self-Injury (NSSI) in a Community Sample of Adolescents, Using Suggested DSM-5 Criteria for a Potential NSSI Disorder. J Abnorm Child Psychol. 2013;41(5):759-773.

21. American Psychiatric Association (APA). DSM-5 Section III - American Psychiatric Association. Available from:l http://www.psych.org/File $\% 20$ Library/Practice/DSM/DSM-5/DSM-5-Section-III.pdf. Accessed December 3, 2013.

22. Plener PL, Fegert JM. Non-suicidal self-injury: state of the art perspective of a proposed new syndrome for DSM V. Child Adolesc Psychiatry Ment Health. 2012;6:9.

22. Moran M. Section III of New Manual Looks to Future. Psychiatric News. May 17, 2013. DOI: 10.1176/appi.pn.2013.5b8

23. Freedman R, Lewis DA, Michels R, et al. The initial field trials of DSM-5: new blooms and old thorns. Am J Psychiatry. 2013; 170(1):1-5.

24. Sher L, Stanley BH. The role of endogenous opioids in the pathophysiology of self-injurious and suicidal behavior. Arch Suicide Res. 2008;12(4):299-308.
25. Stanley B, Sher L, Wilson S, Ekman R, Huang YY, Mann JJ. Non-suicidal self-injurious behavior, endogenous opioids and monoamine neurotransmitters. J Affect Disord. 2010;124(1-2):134-140.

26. Rebecca C. Groschwitz Paul L. Plener. The Neurobiology of Non-suicidal Self-injury (NSSI): A review. Suicidol Online. 2012;3:24-32.

27. Smith NB, Kouros CD, Meuret AE. The Role of Trauma Symptoms in Nonsuicidal Self-Injury. Trauma Violence Abuse. Epub July 22, 2013.

28. Levesque C, Lafontaine M, Bureau J, Cloutier P, Dandurand C. The influence of romantic attachment and intimate partner violence on non-suicidal self-injury in young adults. $J$ Youth Adolesc. 2010;39: 474-483.

29. Connors R. Self-injury in trauma survivors: 1 . Functions and meanings. Am J Orthopsychiatry. 1996;66:197-206.

30. Klonsky ED, Moyer A. Childhood sexual abuse and non-suicidal selfinjury: meta-analysis. Br J Psychiatry. 2008;192(3):166-170.

31. Eysenbach G. Infodemiology and infoveillance tracking online health information and cyberbehavior for public health. Am J Prev Med. 2011;40(5 Suppl 2):S154-S158

32. Eysenbach G. Infodemiology and infoveillance: framework for an emerging set of public health informatics methods to analyze search, communication and publication behavior on the Internet. J Med Internet Res. 2009;11(1):e11.

33. Lewis SP, Heath NL, Michal NJ, Duggan JM. Non-suicidal self-injury, youth, and the Internet: What mental health professionals need to know. Child Adolesc Psychiatry Ment Health. 2012;6(1):13.

34. Lloyd-Richardson E, Perrine N, Dierker L, Kelley ML. Characteristics and functions of non-suicidal self-injury in a community sample of adolescents. Psychol Med. 2007;37:1183-1192.

35. Lewis SP, Heath NL, Sornberger MJ, Arbuthnott AE. Helpful or harmful? An examination of viewers' responses to nonsuicidal selfinjury videos on YouTube. J Adolesc Health. 2012;51(4):380-385.

36. Lewis SP, Rosenrot SA, Messner MA. Seeking validation in unlikely places: the nature of online questions about non-suicidal self-injury. Arch Suicide Res. 2012;16(3):263-272.

37. Whitlock JL, Powers JL, Eckenrode J.The virtual cutting edge: the internet and adolescent self-injury. Dev Psychol. 2006;42(3):407-417.

38. Lewis SP, Baker TG. The possible risks of self-injury web sites: a content analysis. Arch Suicide Res. 2011;15(4):390-396.

39. McSwain S, Lester D, Gunn JF 3rd. Warning signs for suicide in Internet forums. Psychol Rep. 2012;111(1):186-188.

40. Yang AC, Tsai SJ, Yang $\mathrm{CH}$, et al. Suicide and media reporting: a longitudinal and spatial analysis. Soc Psychiatry Psychiatr Epidemiol. 2013;48(3):427-435.

41. Westerlund M, Hadlaczky G, Wasserman D. The representation of suicide on the Internet: implications for clinicians. J Med Internet Res. 2012;14(5):e122.

42. Durkee T, Hadlaczky G, Westerlund M, Carli V. Internet pathways in suicidality: a review of the evidence. Int $J$ Environ Res Public Health 2011;8(10):3938-3952.

43. Ruder TD, Hatch GM, Ampanozi G, Thali MJ, Fischer N. Suicide announcement on Facebook. Crisis. 2011;32(5):280-282.

44. Noll-Hussong M, Lahmann C. Whiplash and Werther effect: the potential of google insights for search for medical research and public health. Fortschr Neurol Psychiatr. 2011;79(6):340-344. German

45. Birbal R, Maharajh HD, Birbal R, et al. Cybersuicide and the adolescent population: challenges of the future? Int $J$ Adolesc Med Health. 2009;21(2):151-159.

46. Hsiung RC. A suicide in an online mental health support group: reactions of the group members, administrative responses, and recommendations. Cyberpsychol Behav. 2007;10(4):495-500.

47. Recupero PR, Harms SE, Noble JM. Googling suicide: surfing for suicide information on the Internet. J Clin Psychiatry. 2008; 69(6):878-888.

48. McCarthy MJ. Internet monitoring of suicide risk in the population. J Affect Disord. 2010;122(3):277-279. 
49. Ginsberg J, Mohebbi MH, Patel RS, Brammer L, Smolinski MS, Brilliant L. Detecting influenza epidemics using search engine query data. Nature. 2009;457(7232):1012-1014.

50. Bragazzi NL. Infodemiology and infoveillance of Multiple Sclerosis in Italy. Mult Scler Int. 2013;924029. doi: 10.1155/2013/924029. Epub August, 20, 2013.

51. Sueki H. Does the volume of Internet searches using suicide-related search terms influence the suicide death rate: data from 2004 to 2009 in Japan. Psychiatry Clin Neurosci. 2011;65(4):392-394.

52. Hagihara A, Miyazaki S, Abe T. Internet suicide searches and the incidence of suicide in young people in Japan. Eur Arch Psychiatry Clin Neurosci. 2012;262(1):39-46.

53. Yang AC, Tsai SJ, Huang NE, Peng CK. Association of Internet search trends with suicide death in Taipei City, Taiwan, 2004-2009. J Affect Disord. 2011;132(1-2):179-184.
54. Page A, Chang SS, Gunnell D. Surveillance of Australian suicidal behaviour using the internet? Aust N Z J Psychiatry. 2011;45(12): 1020-1022.

55. Gunn JF 3rd, Lester D. Using Google Searches on the Internet to monitor suicidal behavior. J Affect Disord. 2013;148(2-3):411-412.

56. Sueki H. Association between deliberate self-harm-related Internet searches and the mental states and lifetime suicidal behaviors of Japanese young adults. Psychiatry Clin Neurosci. 2012;66(5):451-453.

57. (Partial) Autocorrelation Function - Free Statistics Software [webpage on the internet]. Wessa P; 2012. Available from: http://www.wessa.net/ rwasp_autocorrelation.wasp/. Accessed December 3, 2013.

58. Pearson Correlation - Free Statistics Software (Calculator) webpage on the internet. Wessa P; 2012. Available from: http://www.wessa.net/ rwasp_correlation.wasp/. Accessed December 3, 2013.

\section{Publish your work in this journal}

Psychology Research and Behavior Management is an international, peerreviewed, open access journal focusing on the science of psychology and its application in behavior management to develop improved outcomes in the clinical, educational, sports and business arenas. Specific topics covered include: Neuroscience, memory \& decision making; Behavior modification \& management; Clinical applications; Business \& sports performance management; Social and developmental studies; Animal studies. The manuscript management system is completely online and includes a quick and fair peer-review system. Visit http://www.dovepress. com/testimonials.php to read real quotes from published authors. 